\title{
The Human Microbiota in Multiple Myeloma and Proteasome Inhibitors
}

\author{
Omar Alkharabsheh $^{\mathrm{a}}$ M. Hasib Sidiqi ${ }^{\mathrm{b}}$ Mohammed A. Aljamac \\ Morie A. Gertz ${ }^{\mathrm{b}}$ Arthur E. Frankel ${ }^{\mathrm{a}}$ \\ aDivision of Medical Oncology, Mitchell Cancer Institute, University of South Alabama, Mobile, AL, USA; \\ ${ }^{b}$ Division of Hematology, Department of Medicine, Mayo Clinic, Rochester, MN, USA; ' Division of Malignant \\ Hematology, Department of Oncology, McMaster University, Hamilton, ON, Canada
}

\section{Keywords}

Multiple myeloma $\cdot$ Proteasome inhibitors · Microbiota

\begin{abstract}
The gut microbiota plays a significant role in health and disease, including cancer development and treatment. The importance of the gut microbiota in the efficacy and toxicity of novel therapies and immunotherapy is increasingly recognized. Plasma cells in multiple myeloma have the potential to survive in the gastrointestinal tract for long periods of time. The nature of the gut microbiota impacts the degree of antigen stimulation of these cells and may play a role in mutation development and clonal evolution. Furthermore, myeloma therapies such as proteasome inhibitors and alkylating agents, commonly used to treat patients, are frequently associated with gastrointestinal adverse events. Herein we review the gut microbiota and its role in hematopoiesis, pathogenesis of myeloma, and efficacy/toxicity of anti-myeloma therapies.
\end{abstract}

\section{Introduction}

The human microbiota is becoming increasingly important in the era of immune and cellular therapy. The complex and interactive balance between nonpathogenic microbes and our immune system has created a steady state of coexistence. Any disturbance of this balance can lead to morbidity and illnesses. Literature published regarding the gut microbiota in cancer research has increased dramatically since the early 2000 s. In addition to the gut microbiota's role in the promotion and prevention of disease, reports are now emerging of an effect on cancer treatment outcomes [1].

The success of immunotherapy in various malignancies is dependent on several factors, such as the level of antigen expression on the surface of cells (e.g., programmed death ligand-1 expression), the amount of tumor-infiltrating lymphocytes, the tumor mutational burden, somatic aberration, and likely more that will be identified with ongoing research [2].

Within the gut microbiota, both pathogenic and nonpathogenic microbes exist, which are susceptible to change according to the local environment. These microbes are under active surveillance by our immune sys-

\section{KARGER}

() 2019 S. Karger AG, Basel

karger@karger.com

www.karger.com/aha
Omar Alkharabsheh, MD, Assistant Professor

University of South Alabama

1660 Spring Hill Ave.

Mobile, AL 36604 (USA)

E-Mail oalkharabsheh@health.southalabama.edu 
tem, which can be activated and eradicate pathogenic microbes or attack the host cells, particularly the intestinal epithelium, as seen in inflammatory bowel diseases. Therefore, scientists have speculated that active immune system recognition triggered by the presence of nonpathogenic microbes may enhance the efficacy of immunological and cellular therapies. However, this same microbe-dependent immune activation may also play a role in increasing the risk of immune-mediated adverse events [1].

Multiple myeloma is a malignancy of plasma cells. Plasma cells are considered the backbone of the adaptive immune system due to their ability to produce immunoglobulins and fight microbial infections. When plasma cells become clonal by acquiring somatic mutations that alter their function, mainly involving the immunoglobulin heavy chain $(\mathrm{IgVH})$ on chromosome 14, they produce abnormal proteins (monoclonal proteins), which have immunogenic properties that can drive the immune system against the host [3].

The dramatic improvement in multiple myeloma therapy in the last decade, with the introduction of advancedgeneration proteasome inhibitors, immunomodulators, and monoclonal antibodies, has improved outcomes and survival [4]. Several agents exhibit adverse effects on the immune system and the gastrointestinal (GI) tract. For example, diarrhea is a well-established side effect of proteasome inhibitors, and lymphodepletion is a treatment side effect that increases the risk of opportunistic infections [5]. Understanding the correlation between plasma cells, the gut microbiota, and proteasome inhibitors is crucial to improve treatment efficacy and decrease adverse events.

\section{We Are Not Alone, Gut Microbiota!}

Our gut microbiota has a role in our health starting from early life, and its effect goes beyond simple colonization [6]. The large surface area of our GI tract makes it vulnerable to microbial infection. Both innate and adaptive immune systems are present in the lamina propria and the mesenteric lymphatic system, leading to continuous stimulatory signals throughout the GI tract. Therefore, any disturbance in the ecosystem of the microbiota would impact the immune system, which may trigger an inflammatory state. In terms of the composition of the microbiota, multiple types of commensal bacteria exist, with the most common being Firmicuteslike Lactobacillus, Enterococcus, Clostridium, and Bacil- lus. These bacteria represent a spectrum of gram-positive aerobes and anaerobes, as well as others such as Bacteroidetes (gram negative) and Actinobacteria [7]. The anaerobic environment in our GI tract enables bacterial fermentation and the production of metabolites that have immunological activity with the ability to pass into the systemic circulation, such as short-chain fatty acids (SCFAs) [8].

SCFAs, butyrate in particular, have an important regulatory effect on the immune system, including both its innate and adaptive arms [9]. Other SCFAs, such as acetate and propionate, have an effect through the G-protein-coupled receptor, mainly free fatty acid 2 , leading to the activation of different downstream signaling cascades and ultimately the inhibition of histone deacetylase (HDAC) [10]. This pathway contributes to enhancing myelopoiesis and thus to the enhancement of the antibacterial activity of macrophages [11]. An additional effect of SCFAs on the host immune response is the promotion of plasma cells to produce IgA in the GI tract and regulate T-cell differentiation [12].

\section{The Microbiota and Hematopoiesis}

As depicted in Figure 1, SCFAs have several effects on inflammatory cytokines and pathways. SCFAs are produced by the gut microbiota as a result of carbohydrate fermentation. SCFAs have been reported to have an additional effect on the marrow microenvironment through the suppression of T-helper-17 cells (Th-17) and by increasing regulatory $\mathrm{T}$-cell activity, which enhances the steady state of the immune system and allows for appropriate hematopoiesis and marrow function [13]. The main subtype of SCFAs, butyrate, decreases T-cell function in the GI lamina propria [14]. Bacteroides fragilis produces polysaccharide $\mathrm{A}$, which has an anti-inflammatory effect and can migrate to the systemic circulation [15]. One important benefit of a balanced state between the gut microbiota and our immune system is the absorption of important nutrients for hematopoiesis, such as iron and vitamin $\mathrm{B}_{12}$. Hepcidin is an important regulator in iron metabolism and absorption. It is usually increased in an inflammatory state, thus decreasing iron absorption, leading to anemia of chronic disease or inflammation. Additional mechanisms for anemia of chronic disease, whether it is erythropoietin dependent or not, are inflammatory cytokine effects on the marrow microenvironment causing decreased erythropoiesis irrespective of erythropoietin level [16]. 


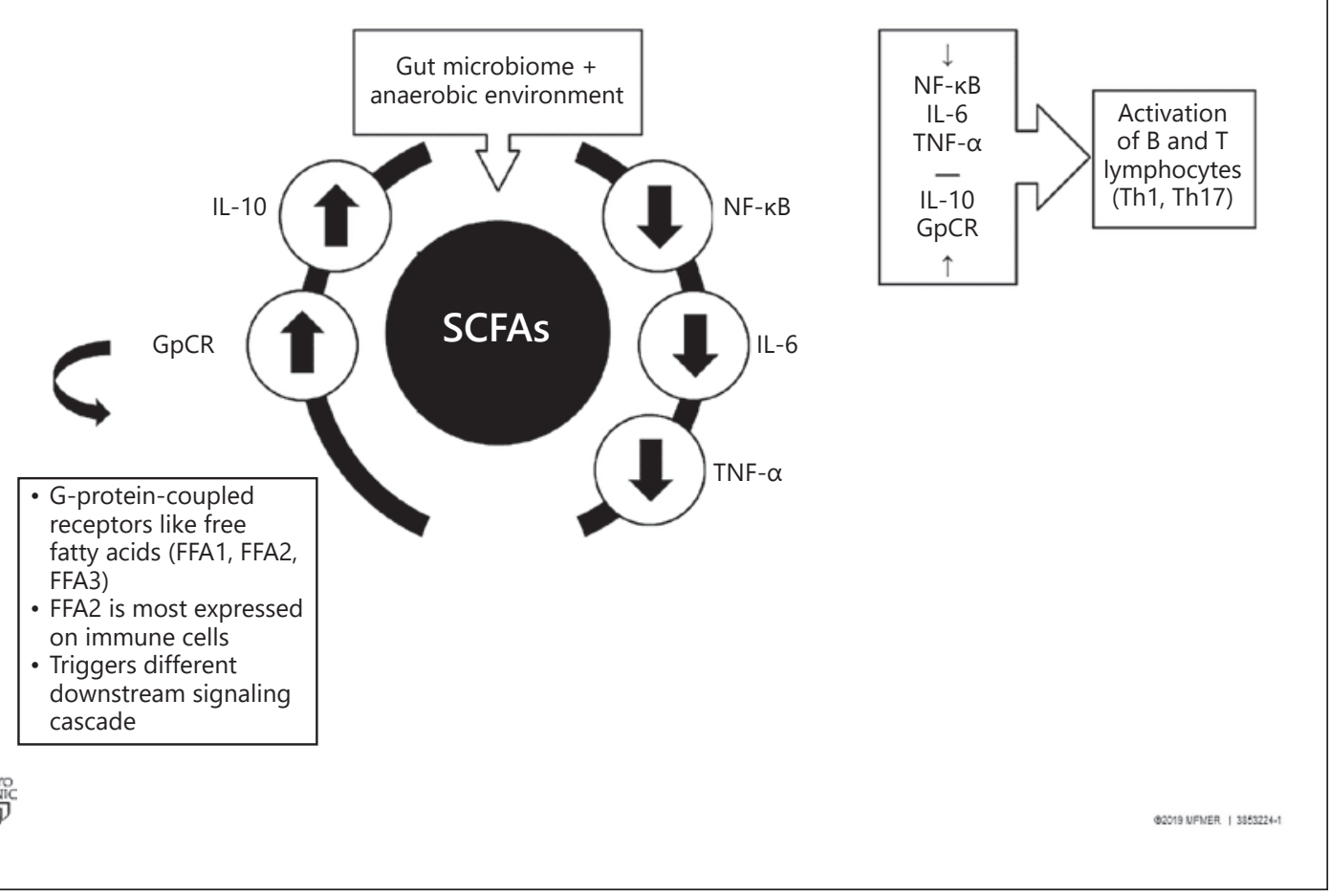

Fig. 1. Effects of short-chain fatty acids on various cytokines and inflammatory pathways. SCFAs, short-chain fatty acids; Th, T-helper cell; NF- $\kappa \mathrm{B}$, nuclear factor kappa-light-chain-enhancer of activated B cells; GPCR, Gprotein-coupled receptor; IL, interleukin; TNF, tumor necrosis factor.

From the blood and marrow transplant literature, we obtain a good understanding about the effects of the gut microbiota in patients receiving therapy for hematological malignancies. Conditioning regimens that include high-dose chemotherapy change the diversity of the gut microbiota (dysbiosis), which may affect the outcome of the engrafted stem cells [13]. The efficacy of allogeneic stem cell transplants depends on the cytotoxic conditioning regimens and the donor cells exhibiting a graft-versus-tumor effect. Previously, we believed that the depth of response correlates with the above two factors. We have learned that more variables are involved in outcomes of marrow transplantation and hematological malignancies, including the marrow microenvironment and complex molecular aberrations resistant to chemotherapy. There is rising evidence of gut microbiota having effects on outcomes of hematological malignancies. In a large retrospective series for patients undergoing allogeneic stem cell transplantation, stool analysis using $16 \mathrm{~s}$ rRNAs showed that higher levels of Eubacterium limosum were associated with decreased risk of relapse/progression [17].

\section{What Is Special about Plasma Cells?}

Our bone marrow environment stores hematopoietic stem cells. The lymphoid stem cells produce lymphoblasts, which differentiate into mature $\mathrm{T}$ and $\mathrm{B}$ lymphocytes. B cells in the setting of antigen activation may undergo terminal differentiation to plasma cells. Plasma cells are responsible for the production of immunoglobulin in response to stimuli or external antigens. The events preceding terminal differentiation determines the type of antibody produced by the activated B cells [18].

The gut microenvironment is rich in immunologic stimuli from both the normal flora and external pathogens. The production of immunoglobulin is partially dependent on T-cell stimulation based on the type of produced immunoglobulin: IgM versus other (IgG, IgA, or IgD) [19]. With the development of clonal plasma cells, immunoglobulin production is dysregulated, leading to the accumulation of monoclonal proteins. Researchers from the University of Oslo discovered that plasma cells can potentially live for decades in the intestinal tract, particularly those lacking expression of CD19 and CD45 [20]. 
Fig. 2. NF- $\mathrm{B}$ pathway and proteasome inhibitors: TNF- $\alpha$ will activate I $\mathrm{B}$ kinase (IKK) which will lead to phosphorylation of $\mathrm{I} \kappa \mathrm{B}$, causing separation of NF- $\kappa \mathrm{B}$ and $\mathrm{I} \kappa \mathrm{B}$ and thus initiation of gene transcription. PIs enhance the activation of NF- $\kappa \mathrm{B}$ by suppressing ubiquitylation of phosphorylated $\mathrm{I} \kappa \mathrm{B}$.

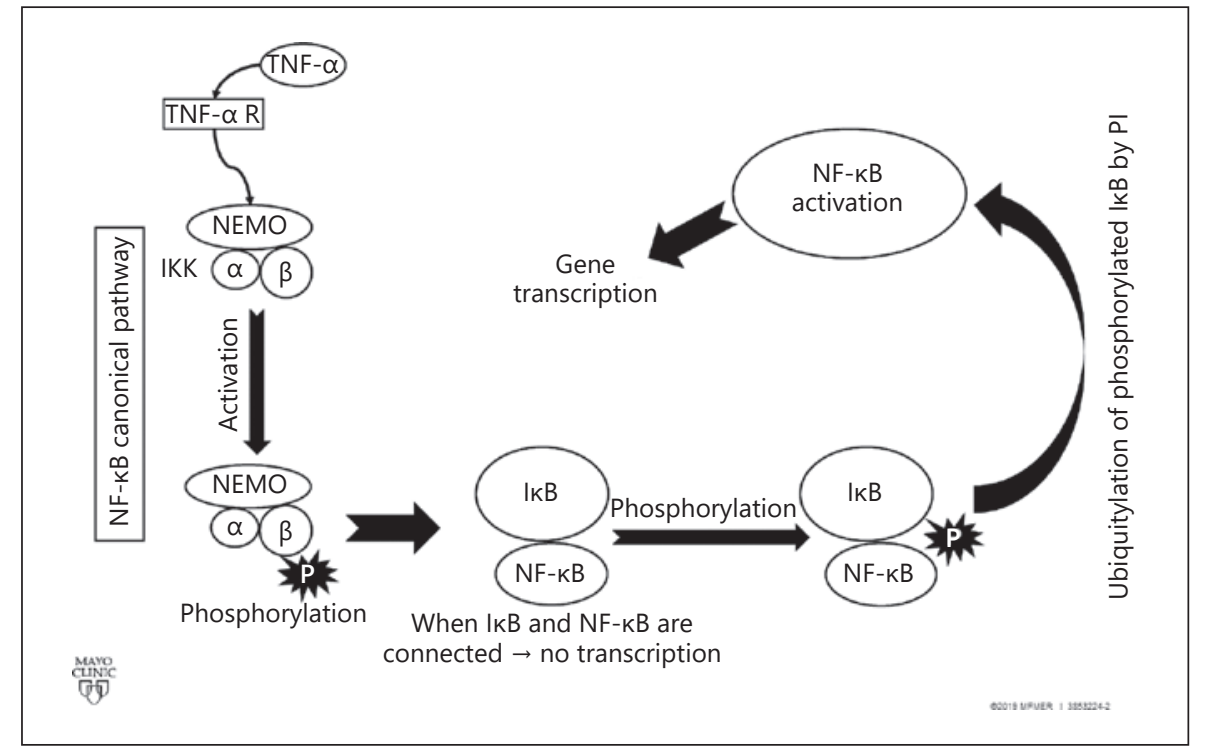

Long-term exposure to stimuli and external antigens increases the chance of genetic mutational development, leading to clonal plasma cells and eventually to plasma cell neoplasms, such as multiple myeloma. Whether the gut microbiota's interaction with our immune system contributes to mutational development is unclear and requires further study.

\section{Effects of Proteasome Inhibitors on the Gastrointestinal Tract}

Proteasome inhibitors (PIs) are considered the backbone of multiple myeloma treatment in the frontline and relapsed refractory settings [21]. Multiple trials have examined first- and second-generation PIs, including oral formulations, and have reported GI toxicity. Based on preclinical data, an increase in tumor necrosis factor alpha (TNF- $\alpha$ ) receptor 1 expression on the intestinal epithelium and the elevated pro-inflammatory cytokine-like TNF- $\alpha$, interleukin (IL)- $1 \beta$, and IL- 6 were proposed as the underlying etiology of GI toxicity, mainly in the form of diarrhea and, rarely, inflammatory colitis [22, 23].

As described previously, dysregulation of the gut microbiota alters our immune system, resulting in an inflammatory state and adverse events. Whether PIs have a direct effect on the gut microbiota or not will need further experimentation and research. We know that PIs inhibit the nuclear factor kappa-light-chain-enhancer of activated $B$ cells $(N F-\kappa B)$ pathway, which has been shown to decrease the risk of acute graft-versus-host disease in the GI tract [24]. Thus, we speculate that the dominant pathway contributing to GI toxicity due to PIs is NF- $\kappa B$ dysregulation. It will be important to ascertain the role of the gut microbiota in the regulation of the NF- $\kappa \mathrm{B}$ pathway and whether this impacts the GI toxicity seen with PIs.

Some reports demonstrate that gut microbiota products, such as SCFAs, suppress NF- $\kappa B$ and inflammatory cytokines, which can augment the PI effect on the GI tract. However, the effects of PIs on TNF- $\alpha$ and its receptors would still produce GI adverse events in the form of diarrhea, which has been reported in multiple trials [25].

\section{Myeloma Treatments and Gut Microbiota Balance}

Cyclophosphamide is an alkylator that has been used in various triplet regimens for myeloma [21]. In preclinical studies, mouse models with implanted tumor cells demonstrated resistance to cyclophosphamide if previously treated with antibiotics to achieve a germ-free gut microenvironment. Therefore, researchers proposed that the presence of the gut microbiota increases the sensitivity of tumor cells to cyclophosphamide via Th17 and Th1 cells and immune responses [26].

Autologous stem cell transplant is considered a standard of care in treating multiple myeloma patients who are fit and eligible [21]. During the critical cytopenia period until engraftment, patients are usually treated with multiple antimicrobial prophylactic agents and are at 
high risk of opportunistic infections from the GI tract. Prior to this phase of transplant, the patient receives highdose conditioning chemotherapy that alters the biology and normal flora of the GI epithelium and thus leads to the monodominance of pathogenic microbes such as Enterobacteriaceae [27]. In allogeneic stem cell transplant, E. limosum has been associated with a decreased risk of relapse and progression in several hematologic malignancies. A study included 541 patients (11\% multiple myeloma) who underwent stool sample analysis for $16 \mathrm{~s}$ rRNAs. At 2 years, a higher diversity of bacterial groups indicated better outcomes: hazard ratio $0.29(0.12-0.67)$, $p=0.004$ [17].

In myeloma treatment, the patterns of progression suggest that gut microbiota dysregulation impacts treatment outcomes and patterns of resistance to chemotherapy. This has also been observed with several anti-cancer agents, including immune checkpoint inhibitors [26, 28]. Additional reports by Calcinotto et al. [29] demonstrated a more direct correlation between the gut microbiota and cancer. Th17 has been associated with disease progression and more clonal expansion of plasma cells in the bone marrow. This can be driven by the stimulation of the gut microbiota to Th17. However, the exact role of Th17 and its product IL-17 and their effect on tumor cells have not been consistent in the literature, with reports of both progression and regression of clonal plasma cells [30, 31]. Calcinotto et al. [29] reported that higher levels of IL-17 in bone marrow increase the risk of progression of smoldering myeloma to multiple myeloma; thus, inhibiting IL-17 can be a target for smoldering myeloma treatment. Further research has correlated SCFAs, mainly butyrate, produced by Eubacterium hallii and Faecalibacterium prausnitzii, are associated with minimal residual disease negativity at the end of induction therapy for multiple myeloma [32].

\section{Discussion}

Activation of the NF- $\kappa \mathrm{B}$ pathway promotes cell proliferation, tumor progression, and the suppression of apoptosis [33]. SCFAs, which are produced by the fermentation of carbohydrates in the intestinal tract by the gut microbiota, have anti-inflammatory effects [34]. The suppression of the pro-inflammatory cytokines by SCFAs occurs through the NF- $\kappa \mathrm{B}$ pathway, histone deacetylase inhibition, and TNF- $\alpha$ [10]. The inhibition of the NF- $\kappa B$ pathway is thought to occur via the suppression of the

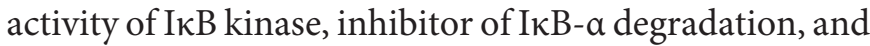
NF- $\kappa B$ separation from phosphorylated I B (Fig. 2) [35].
The underlying pathophysiology of GI toxicity of PIs is poorly understood. Stansborough et al. [22] proposed that PIs cause proinflammatory cytokine release, the disruption of microbiota balance, or possible autonomic neuropathy.

Since both PIs and SCFAs inhibit the NF- $\kappa$ B pathway and PI-induced diarrhea is still recorded in patients with multiple myeloma, more variables have to be examined to better understand the pathophysiology of PI-induced diarrhea, especially with data presented at the American Society of Hematology meeting in December 2018 showing minimal residual disease negativity at the end of induction associated with SCFAs (butyrate) [32].

We speculate that fecal microbiota transplantation (FMT) can increase the SCFA levels in the intestinal tract and thus decrease the GI side effects of PIs. As the field of FMT is evolving in treating several diseases, such as refractory Clostridium difficile infections, GI graft-versushost disease for blood and marrow transplant patients, or immune checkpoint-mediated colitis refractory to glucocorticoids, experimenting with FMT in PI-induced diarrhea would provide a sound scientific basis.

\section{Disclosure Statement}

Dr. Gertz reports grants and personal fees from Spectrum; personal fees from Ionis/Akcea, Alnylam, Prothena, Celgene, Janssen, Annexon, Appellis, Amgen, Medscape, Physicians Education Resource, Data Safety Monitoring Board of Abbvie, and Research to Practice; speaker fees from Teva, Johnson and Johnson, Medscape, and DAVA oncology; and has served on the Advisory Board of Pharmacyclics and Proclara outside the submitted work.

He has also received funding from the Educational Program Development i3Health; royalties from Springer Publishing Grant Funding Amyloidosis Foundation; and a grant from the International Waldenstrom Foundation (NCI SPORE MM SPORE 5P50 CA186781-04). All other authors declare no competing financial interests.

\section{Funding Sources}

This review article received no funding.

\section{Author Contributions}

O.A. wrote the initial draft and approved the final version of the paper. M.H.S., M.A.A., M.A.G., and A.E.F. revised the manuscript critically, and approved the final version of the paper. 


\section{References}

1 Routy B, Le Chatelier E, Derosa L, Duong CP, Alou MT, Daillère R, et al. Gut microbiome influences efficacy of PD-1-based immunotherapy against epithelial tumors. Science. 2018 Jan;359(6371):91-7.

2 Jenkins RW, Thummalapalli R, Carter J, Cañadas I, Barbie DA. Molecular and Genomic Determinants of Response to Immune Checkpoint Inhibition in Cancer. Annu Rev Med. 2018 Jan;69(1):333-47.

3 Kuehl WM, Bergsagel PL. Multiple myeloma: evolving genetic events and host interactions. Nat Rev Cancer. 2002 Mar;2(3):175-87.

4 Moreau P. How I treat myeloma with new agents. Blood. 2017 Sep;130(13):1507-13.

5 Manasanch EE, Orlowski RZ. Proteasome inhibitors in cancer therapy. Nat Rev Clin Oncol. 2017 Jul;14(7):417-33.

6 Cani PD. Human gut microbiome: hopes, threats and promises. Gut. 2018 Sep;67(9): 1716-25.

7 Qin J, Li R, Raes J, Arumugam M, Burgdorf KS, Manichanh C, et al.; MetaHIT Consortium. A human gut microbial gene catalogue established by metagenomic sequencing. $\mathrm{Na}-$ ture. 2010 Mar;464(7285):59-65

8 Tan J, McKenzie C, Potamitis M, Thorburn AN, Mackay CR, Macia L. The role of shortchain fatty acids in health and disease. Adv Immunol. 2014;121:91-119.

9 Gonçalves P, Araújo JR, Di Santo JP. A CrossTalk Between Microbiota-Derived ShortChain Fatty Acids and the Host Mucosal Immune System Regulates Intestinal Homeostasis and Inflammatory Bowel Disease. Inflamm Bowel Dis. 2018 Feb;24(3):558-72.

10 Li M, van Esch BC, Wagenaar GT, Garssen J, Folkerts G, Henricks PA. Pro- and anti-inflammatory effects of short chain fatty acids on immune and endothelial cells. Eur J Pharmacol. 2018 Jul;831:52-9.

11 Schulthess J, Pandey S, Capitani M, Rue-Albrecht KC, Arnold I, Franchini F, et al. The Short Chain Fatty Acid Butyrate Imprints an Antimicrobial Program in Macrophages. Immunity. 2019 Feb;50(2):432-445.e7.

12 Sun M, Wu W, Chen L, Yang W, Huang X, $\mathrm{Ma} \mathrm{C}$, et al. Microbiota-derived short-chain fatty acids promote Th1 cell IL-10 production to maintain intestinal homeostasis. Nat Commun. 2018 Sep;9(1):3555.

13 Manzo VE, Bhatt AS. The human microbiome in hematopoiesis and hematologic disorders. Blood. 2015 Jul;126(3):311-8.
14 Zimmerman MA, Singh N, Martin PM, Thangaraju M, Ganapathy V, Waller JL, et al. Butyrate suppresses colonic inflammation through HDAC1-dependent Fas upregulation and Fas-mediated apoptosis of T cells. Am J Physiol Gastrointest Liver Physiol. 2012 Jun;302(12):G1405-15.

15 Mazmanian SK, Round JL, Kasper DL. A microbial symbiosis factor prevents intestinal inflammatory disease. Nature. 2008 May; 453(7195):620-5.

16 Theurl I, Mattle V, Seifert M, Mariani M, Marth C, Weiss G. Dysregulated monocyte iron homeostasis and erythropoietin formation in patients with anemia of chronic disease. Blood. 2006 May;107(10):4142-8.

17 Peled JU, Devlin SM, Staffas A, Lumish M, Khanin R, Littmann ER, et al. Intestinal Microbiota and Relapse After HematopoieticCell Transplantation. J Clin Oncol. 2017 May; 35(15):1650-9.

18 Chen-Kiang S. Biology of plasma cells. Best Pract Res Clin Haematol. 2005;18(4):493507.

19 MacLennan IC. Germinal centers. Annu Rev Immunol. 1994;12(1):117-39.

20 Landsverk OJ, Snir O, Casado RB, Richter L, Mold JE, Réu P, et al. Antibody-secreting plasma cells persist for decades in human intestine. J Exp Med. 2017 Feb;214(2):309-17.

21 National Comprehensive Cancer Network. NCCN Guidelines for Patients: Multiple Myeloma (Version 2.2019). Available from: https://www.nccn.org/patients/guidelines/ myeloma/4/.

22 Stansborough RL, Gibson RJ. Proteasome inhibitor-induced gastrointestinal toxicity. Curr Opin Support Palliat Care. 2017 Jun; 11(2):133-7.

23 Sun K, Wilkins DE, Anver MR, Sayers TJ, Panoskaltsis-Mortari A, Blazar BR, et al. Differential effects of proteasome inhibition by bortezomib on murine acute graft-versushost disease (GVHD): delayed administration of bortezomib results in increased GVHDdependent gastrointestinal toxicity. Blood. 2005 Nov;106(9):3293-9.

24 Al-Homsi AS, Feng Y, Duffner U, Al Malki MM, Goodyke A, Cole K, et al. Bortezomib for the prevention and treatment of graft-versus-host disease after allogeneic hematopoietic stem cell transplantation. Exp Hematol. 2016 Sep;44(9):771-7.
25 Kumar S, Moreau P, Hari P, Mateos MV, Ludwig $\mathrm{H}$, Shustik C, et al. Management of adverse events associated with ixazomib plus lenalidomide/dexamethasone in relapsed/refractory multiple myeloma. Br J Haematol. 2017 Aug;178(4):571-82.

26 Viaud S, Saccheri F, Mignot G, Yamazaki T, Daillère R, Hannani D, et al. The intestinal microbiota modulates the anticancer immune effects of cyclophosphamide. Science. 2013 Nov;342(6161):971-6.

27 Andermann TM, Peled JU, Ho C, Reddy P, Riches M, Storb R, et al.; Blood and Marrow Transplant Clinical Trials Network. The Microbiome and Hematopoietic Cell Transplantation: Past, Present, and Future. Biol Blood Marrow Transplant. 2018 Jul;24(7):1322-40.

28 Yi M, Yu S, Qin S, Liu Q, Xu H, Zhao W, et al. Gut microbiome modulates efficacy of immune checkpoint inhibitors. J Hematol Oncol. 2018 Mar;11(1):47-47.

29 Calcinotto A, Brevi A, Chesi M, Ferrarese R, Garcia Perez L, Grioni M, et al. Microbiotadriven interleukin-17-producing cells and eosinophils synergize to accelerate multiple myeloma progression. Nat Commun. 2018 Dec; 9(1):4832.

30 Muranski P, Restifo NP. Essentials of Th17 cell commitment and plasticity. Blood. 2013 Mar;121(13):2402-14.

31 McAllister F, Bailey JM, Alsina J, Nirschl CJ, Sharma R, Fan H, et al. Oncogenic Kras activates a hematopoietic-to-epithelial IL-17 signaling axis in preinvasive pancreatic neoplasia. Cancer Cell. 2014 May;25(5):621-37.

32 Pianko MJ, et al. Intestinal Microbiota Composition Is Associated with Minimal Residual Disease Negativity in Patients with Multiple Myeloma. Blood. 2018;132(Suppl 1):3167.

33 Xia Y, Shen S, Verma IM. NF- $\kappa B$, an active player in human cancers. Cancer Immunol Res. 2014 Sep;2(9):823-30.

34 Tedelind S, Westberg F, Kjerrulf M, Vidal A. Anti-inflammatory properties of the shortchain fatty acids acetate and propionate: a study with relevance to inflammatory bowel disease. World J Gastroenterol. 2007 May; 13(20):2826-32.

35 Németh ZH, Wong HR, Odoms K, Deitch EA, Szabó C, Vizi ES, et al. Proteasome inhibitors induce inhibitory kappa B (I kappa B) kinase activation, I kappa B alpha degradation, and nuclear factor kappa B activation in HT-29 cells. Mol Pharmacol. 2004 Feb;65(2): 342-9. 